\title{
Goûter la forme Le krti, une expérience de la forme (Inde du Sud)
}

\section{Fabrice Contri}

\section{(2) OpenEdition}

1 Journals

\section{Édition électronique}

URL : http://journals.openedition.org/ethnomusicologie/466

ISSN : 2235-7688

\section{Éditeur}

ADEM - Ateliers d'ethnomusicologie

Édition imprimée

Date de publication : 1 janvier 2004

Pagination : 241-259

ISBN : 2-8257-0910-7

ISSN : 1662-372X

\section{Référence électronique}

Fabrice Contri, " Goûter la forme Le krti, une expérience de la forme (Inde du Sud) », Cahiers

d'ethnomusicologie [En ligne], 17 | 2004, mis en ligne le 13 janvier 2012, consulté le 01 mai 2019. URL http://journals.openedition.org/ethnomusicologie/466

Ce document a été généré automatiquement le 1 mai 2019.

Tous droits réservés 


\title{
Goûter la forme Le $\mathrm{kṛt}^{1}{ }^{1}$, une expérience de la forme (Inde du Sud)
}

\author{
Fabrice Contri
}

\section{Kanniyākumari² (le mensonge de la forme?)}

1 M'asseoir à la pointe sud de l'Inde. Tout en bas de cet immense triangle de terre, la tête tournée vers l'équateur. Seul. Il n'y a plus de place que pour un seul homme, pensais-je, en regardant la carte de l'atlas géographique, lorsque j'étais enfant. Seul sur cet étroit promontoire et derrière moi tout le sous-continent, puis l'Asie et des milliards d'êtres. Trois océans se rencontrent en ce point; trois, comme les trois formes de la Trimūrti, comme les trois compositeurs de la «Trinité carnatique ${ }^{3}{ }_{1 . . .}$

2 Lorsque, quelques années plus tard, je me rendis à Kanniyākumari, devenu adulte, la pointe océanienne de l'Inde avait sans doute dû être grignotée par je ne sais quel exotique rongeur - peutêtre l'avide souris de Ganapati": je ne vis qu'une longue bande de sable et d'eau, une côte qui, loin de piquer l'océan, semblait au contraire l'absorber, l'enlacer avec douceur telle une anse qui aurait avalé aussi mon rêve d'enfant.

3 C'est donc que la forme m'avait menti. Il y avait en cette carte, malgré la finesse de ses lignes, la précision millimétrique de son échelle, une manière trompeuse de transcrire la réalité. Non, ce n'était peut-être pas la forme, mais plutôt ses contours et mon regard aussi. Car la forme va au-delà de ces simples limites, je le comprenais, de ces frontières que le géographe peut réduire et dessiner. Elle n'est pas uniquement une surface, une figure, une apparence: elle demeure inséparable de son contenu, de ce qui fut à sa source, de ce qui la nourrit, de tout ce qui en donnera la saveur. La goûter, c'est aussi en faire l'expérience.

4 J'avais ma carte, je marchais sur le sol indien, à l'intérieur de sa chaleur moite, de ses sons, de ses odeurs; j'étais au cœeur du fruit. 


\section{Bhakti et musique}

5 La forme dans l'hindouisme, et plus particulièrement au sein de la bhakti ${ }^{5}$, est d'abord ce que l'on perçoit, ce que l'on touche et ce par quoi l'on parvient à être touché. Les sens comme l'esprit humains ont besoin de ce support et de ce moyen pour communiquer. Ainsi les dieux rencontrent-ils les hommes par le biais de leur matérialité. Les idoles expriment leur présence. Objets sacrés, elles ne se satisfont cependant pas d'être adorées, louées avec une vénérable distance. Pour être atteintes, et qu'elles puissent en retour dialoguer avec leurs dévots, elles doivent aussi être « pratiquées».

Un bhakta ne se contente pas d'adorer son dieu par des mots et des rites, il n'est pas plus satisfait par la connaissance de la théologie; il a besoin de le posséder et d'être possédé par lui. Il a besoin aussi de chanter, de faire de la poésie, de la peinture, de la sculpture, d'ériger des lieux de prière; il désire rendre son dieu présent, l'incarner de toutes les façons possibles. Dans la bhakti, tous les arts deviennent une voie d'accès à l'extase, un moyen de posséder et d'être possédé (Rāmānujan 1999, 12).

6 De même que prolifèrent les représentations de la divinité, se multiplient tous les autres supports de son exaltation et de sa louange, d'où l'extraordinaire effervescence de ses modes d'expression, et donc des arts. L'œuvre d'art résonne comme un merveilleux appel aux sens qu'elle ravit, captive à travers ce foisonnement. Sa pratique permet aussi, tout comme celle des idoles, une relation privilégiée de l'être et de l'objet.

Le mot kalā (art) tout comme kalam ${ }^{7}$ renvoie étymologiquement à $k a$ - qui est aussi karana (l'action) - et à la - quiest aussi layam $^{8}$ (la dissolution). Action et dissolution: tels sont les deux éléments fondamentaux de l'art. Lorsque l'artiste crée, quelque chose de lui se dissout dans son œuvre, objet de son action, et fait que nous sommes à notre tour attirés par elle 9 .

7 L'amateur d'art ou le dévot de tel ou tel dieu en quête d'une expression plus complète de son émerveillement, de sa foi, de son amour, ne peut demeurer lui aussi distant de ce qui l'attire. Le terme amour se situe bien au cœur de la bhakti ${ }^{10}$, quête d'une union mystique. Les poètes de la bhakti chantent inlassablement la forme divine qu'ils ont choisi d'adorer ( ișta devata $\left.\bar{a}^{11}\right)$ et leurs propres sentiments à son égard. Le bhakta, même lorsqu'il est poète ou musicien,reste avant tout un dévot face à son dieu.

Pourquoi abandonner femme et enfants pour aller se retirer dans la forêt où vivent les ascètes,

Pourquoi observer des rites religieux et des règles sévères, devenir si maigre après tant de jeûnes,

Ne suffit-il pas de se rappeler le nom divin avec ferveur et une totale dévotion ? (Purandara Dāsa: 1985).

Le nom synthétise la forme, le chanter, le répéter c'est aussi le faire exister.

Tu es le socle de mon existence, le fruit de ma pénitence ! Oh dieu aux yeux de lotus [le compositeur, Tyāgarāja, s'adresse à Rāma ${ }^{12}$ ], le plus glorieux des rois parmi les rois, lumière de ma vue, parfum que je respire ! Tu es la Personnification des lettres mystiques que je chante [les lettres du nom Rāma]. Tu es aussi les fleurs de mes offrandes (Tyāgarāja 1995:549).

9 A chaque répétition du nom, à chacun de ses chants, l'image divine semble absorber un peu plus l'amour du fidèle, prendre corps en lui. Le nom de Rāma, comme la beauté de ses aspects, ne cesse de charmer ses dévots et parmi eux Tyāgarāja (1767-1847), le plus 
célèbre compositeur de l'Inde du Sud. «La musique est mouvement ${ }^{13}$, elle anime le sentiment que le nom porte en lui» ${ }^{14}$.

Fig. 1 : Tyāgarāja, dévot de Rāma

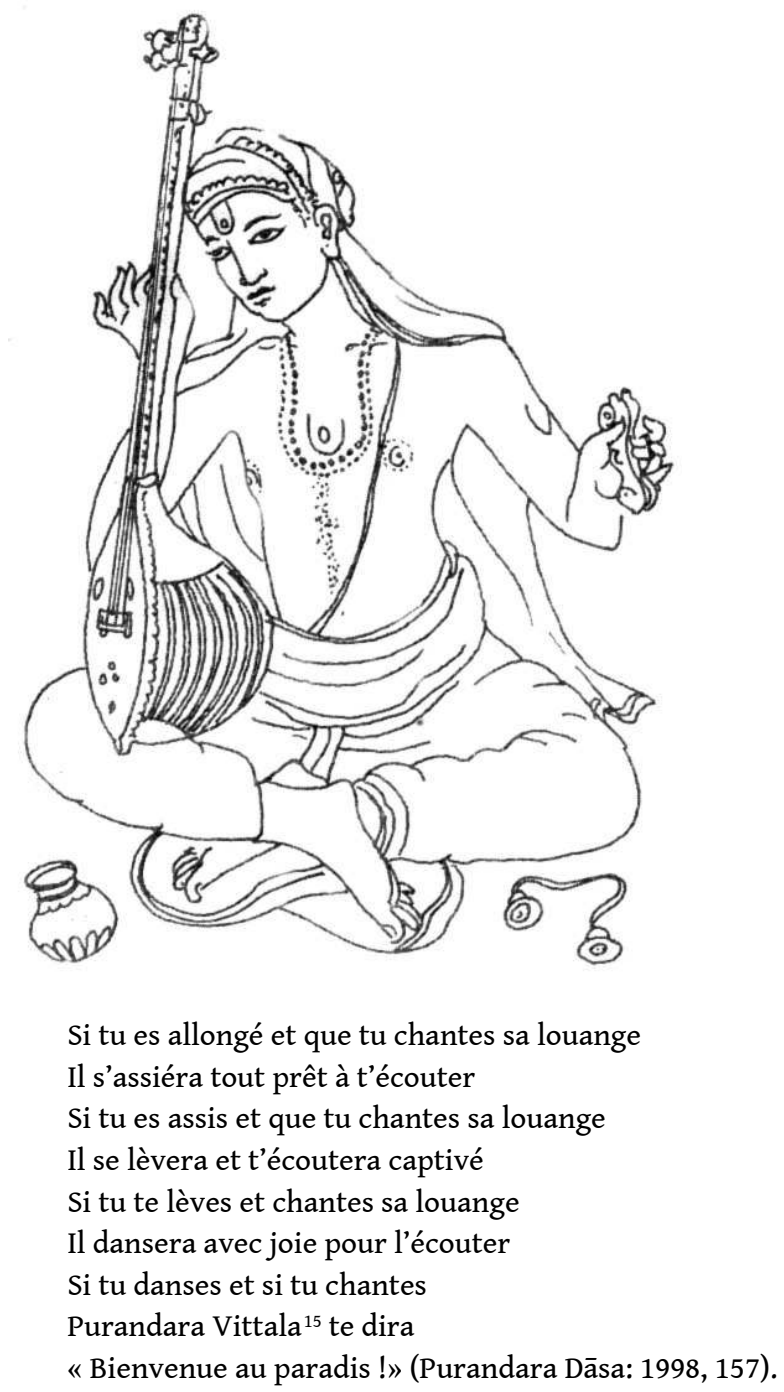

La musique possède en outre cette suprême qualité de pouvoir être interprétée et, en ce sens, toujours actualisable, toujours renouvelée. Car la musique pour exister, pour prendre forme, c'est-à-dire entrer dans le domaine de l'audible, du sensible, doit aussi être vécue. «Connue», s'il ne recelait une connotation proprement biblique, serait sans doute un terme plus opportun, puisque dans ce contexte musical, comme dans la relation du fidèle avec son dieu, il s'agit d'une union, d'une connaissance subtile qui passe préalablement par le prisme de la matérialité de l'être.

\section{Le krti (nommer de la forme)}

Dans quelle intention t'es-tu incarné en Rāma? Est-ce juste pour combattre les rākșasa ${ }^{16}$ ou pour régner sur le royaume d'Ayōdhya ? Ô Rāghava ${ }^{17}$ ! N'agis-tu que par désir de converser avec les yōgi et les ascètes ou de prêter une main secourable aux dévots immergés dans l'océan des plaisirs matériels ? C'est peut-être aussi par compassion pour Tyāgarāja, pour lui accorder tes dons, charmé par les myriades de 
chants qu'il a composés» (Tyāgarāja 1995: 290).

Les compositions $\left(k_{r t i}{ }^{18}\right)$ de Tyāgarāja sont «pleines de jus, comme le raisin»

(Sundaran 1987: 36).

11 Le krti domine depuis plus de trois siècles le répertoire carnatique. Il a été la prédilection des trois plus célèbres compositeurs de la musique carnatique, trois musiciens et poètes, mais également trois grands saints, trois grands bhakta. Parmi eux, Tyāgarāja, « prince de la bhakti» (Tara Bālagopal 1998: 57), ardent dévot de Rāma, figure comme le plus spontané, le plus jaillissant, le plus accessible aussi.

Est-il possible même pour Brahmā, Indra et les autres dieux de décrire ton irrésistible beauté [...] ? Je bénis tes pieds, ô Rāma ! (Tyāgarāja 1995: 96). Comment puis-je satisfaire l'ardent désir de t'étreindre? Puis-je peindre les charmes incomparables de ta personne ? [...] Ta parole est la plus douce des paroles. [...] Ton intelligence brille au-dessus de toutes (Tyāgarāja 1995: 187). Ô Rāma! [...] laissenous contenter notre regard par la captivante beauté de ta forme, ta démarche gracieuse, les reflets d'émeraude de ton corps, les tresses ravissantes de ton front [...], les colliers d'or qui ornent ta poitrine. [...] Accorde-nous le privilège, la bénédiction, l'immense fortune d'admirer ce spectacle sans pareil (Tyāgarāja 1981: 226).

L'incommensurable beauté de Rāma - sa grâce spirituelle et physique - inspira chacun des kṛti composés par Tyāgarāja. « Lorsque Tyāgarāja dit à Rāma, "tu es mon ishta devatā", c'est bien l'insurpassable beauté de ce Syāmasundara ${ }^{19}$ qui lui fait préférer cet aspect du divin» (V. Rāghavan 1981: 138).

Les textes des krti sont ainsi essentiellement des descriptions poétiques de la forme divine adorée mais aussi des différents sentiments qui imprègnent et inspirent le dévot. Le compositeur du krti, qui crée le plus souvent ses poèmes (tel fut toujours le cas de Tyāgarāja ${ }^{20}$ ), exprime les qualités de son ișța devatā et la relation d'amour qu'il entretient avec elle. Le choix initial de l'ișta devatā génère les inspirations créatrices et dicte l'orientation - thématique, formelle et stylistique - des œuvres futures ${ }^{21}$.

La continuelle mémoire du nom de Rāma rend clairement présent dans l'esprit la forme divine et l'emplit de dévotion (Tyāgarāja 1995: 549).

La musique n'est pas uniquement une voie par excellence, « la plus plaisante des ascèses», parce qu'elle s'adresse à tous et touche tous les hommes, par sa simplicité, les émotions qu'elle fait spontanément jaillir en eux, mais aussi parce qu'elle est animation de l'esprit et du corps. Ainsi représente-t-elle l'un des plus efficaces yogas. Le dévot musicien trouve dans le krti un support permanent pour signifier poétiquement et musicalement sa foi, il lui permet de la diriger vers le but suprême: il est une expérience, un guide. Avant de devenir une œuvre d'art, le krti sert donc la bhakti; sa forme, son langage, sont d'abord destinés à cette fonction. Ainsi l'œuvre musicale vaut-elle avant tout pour la hauteur spirituelle de son inspiration, sa capacité à transmettre l'élan dévotionnel qui en fut à la source.

Le centre de l'existence de Tyāgarāja et l'idéal de ses aspirations a été, à chaque souffle, l'expérience de la bhakti de Rāma afin de parvenir à la vision suprême de son ishta devatā. Dans beaucoup de ses chants, son désir s'exprime avec éloquence. Ses œuvres apparaissent remarquables non seulement sur le plan musical mais aussi sur celui de la spiritualité. C'est pour cette raison que ses compositions, comme l' $\bar{a} t \operatorname{tman}^{22}$, perdurent (Krishnamūrthy 2000: 3). 


\section{Les gōpuram ${ }^{23}$ du temple de Madurai ${ }^{24}$ (ressentir la forme)}

15 L'Inde fourmille de formes, peut-être davantage que toute autre contrée au monde: celles de ses millions d'êtres humains, de ses cultures mêlées, celles de ses villes grouillantes - où la vache, le brâhmane, l'homme d'affaire, le mendiant, se croisent dans ses rues inextricables - mais celles surtout de ses divinités qui s'agglutinent, sur la structure de pierre, de métal et de bois de ses temples.

16 L'Inde donne à voir, entendre, sentir, toucher, à satiété. Les gopuram dravidiens - gigantesques montagnes bigarrées, assaillies d'irréels corps humains et animaux - manifestent jusqu'au vertige la divinité à travers cette exubérante sensualité. C'est en effet, d'abord, chacun de nos sens qui semble s'y accrocher.

\section{La partition, la composition (germes de la forme)}

17 La partition - la composition écrite - représente l'état minimal de l'œuvre. Elle est au musicien ce que la carte est au voyageur, c'est-à-dire une représentation schématique d'un itinéraire à découvrir, à parcourir, à occuper.

Même si la notation semble parfaite, elle ne permet pas de saisir l'expression [de l'œuvre] - l'équilibre entre le sentiment du rāga et celui du texte. En fait l'imagination du chanteur lorsqu'il interprète la composition est essentielle afin de lui donner une forme aboutie et d'en faire ressortir toutes les finesses (T. K. Govinda Rao 1997:XV).

18 Trace du geste spontané d'un poète et musicien d'exception envers son dieu, la composition (l'œuvre notée ou fixée dans la mémoire) possède également un caractère sacré. Elle constitue, au sein de la tradition carnatique, le socle de l'interprétation et de la création dont elle garantit, sinon l'excellence, du moins la vénérabilité.

19 La partition se situe donc comme un objet intermédiaire, le « témoin», entre la dimension impalpable de l'inspiration créatrice (principalement la foi) et la réalité sonore de l'œuvre musicale. Au sein du répertoire carnatique, elle ne révèle souvent que de fort loin, en tant que squelette de la forme, l'œuvre musicale finale qui est la forme vécue, exprimée. Il semble y avoir ici le même écart que celui qui sépare la carte du pays, la biographie de la vie effective (différence d'autant plus forte qu'il s'agit, dans ce cas, d'œuvres et de vies de personnages saints).

Une composition musicale peut être comparée à une construction architecturale. Les svara $^{25}$ sont à la mélodie ce que les briques sont au bâtiment. Les différentes oscillations mélodiques qui relient entre eux les svara créent un effet d'ornementation. Elles peuvent être comparées au ciment qui unit les briques et également aux décorations extérieures d'un bâtiment. De même qu'il existe de petits et de grands bâtiments, il y a de courtes et de longues compositions (P. Sambamoorthy 1976: 30). 
Fig. 2: Habiller, décorer, pratiquer les idoles, Dārāsuram, Tamil Nādu

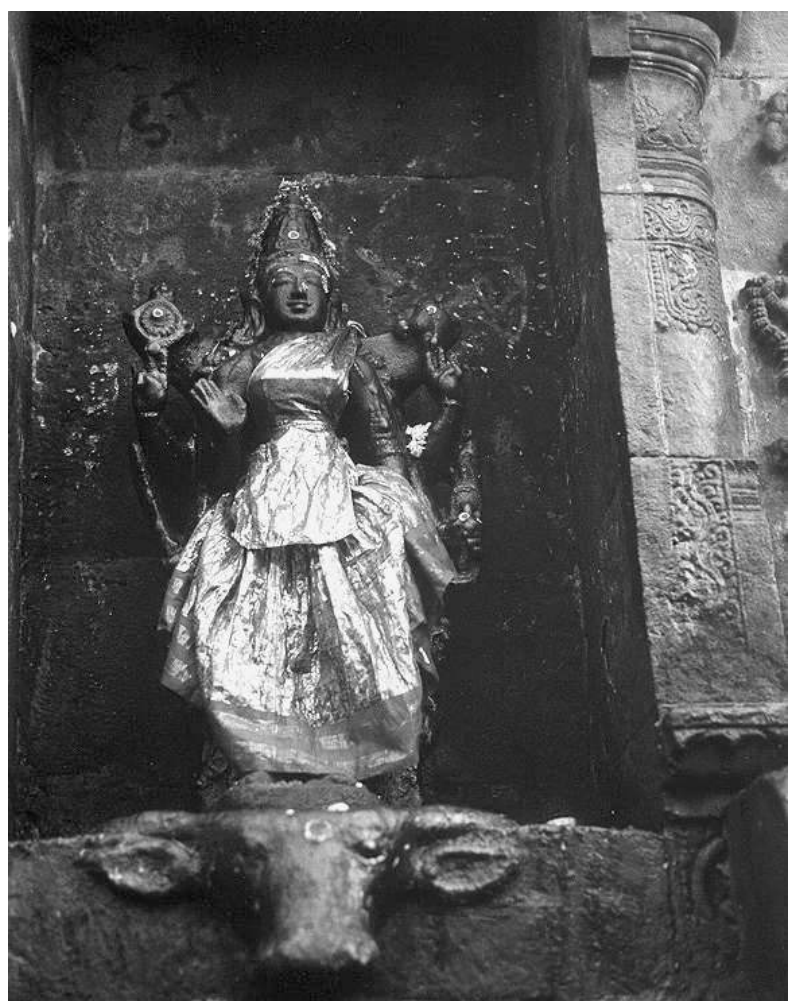

Photo: Fabrice Contri, 2000

Fig. 3: Les Gopuram du temple de Madurai, Tamil Nādu

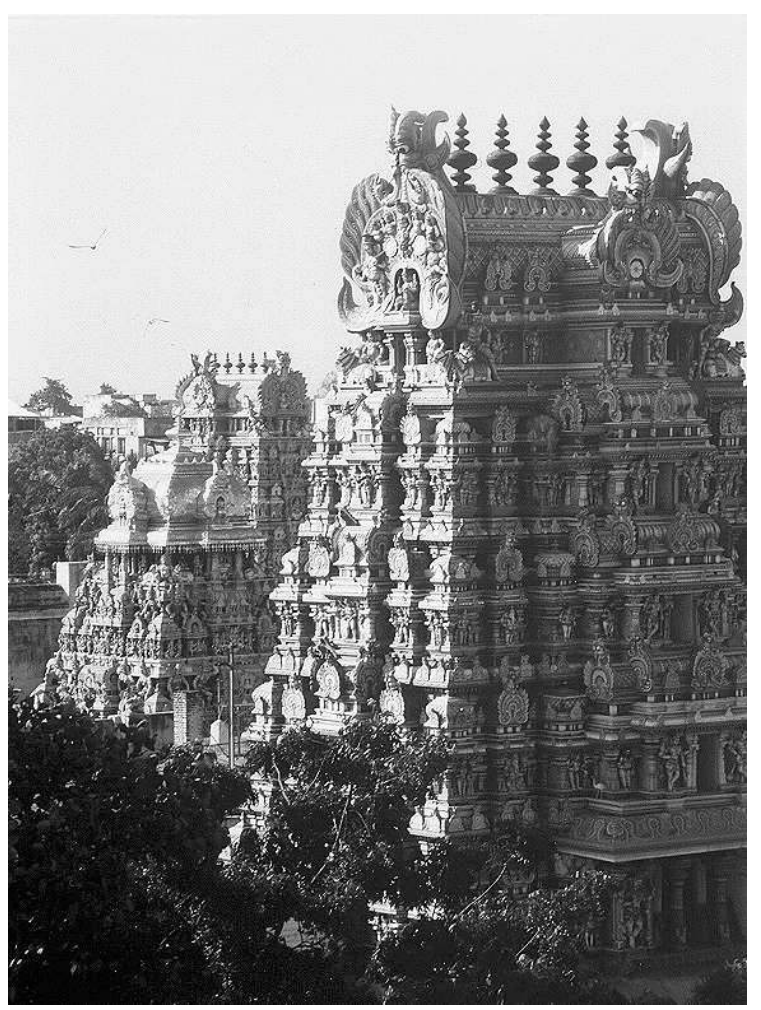

Photo: Fabrice Contri, 2003 
métaphore, peu convaincante sur le plan poétique et musicologique, apparaît cependant symptomatique d'une certaine conception de l'invention formelle dans le contexte compositionnel carnatique. Il semble que dans ce cadre, le génie individuel cherche peu à « innover», si l'on entend du moins par forme musicale « un ensemble de réalités sonores que l'auteur organise de façon immuable» (Eco 1965: 16). L'invention musicale opère alors essentiellement par le biais de l'interprétation, celle-ci pouvant être définie comme un mouvement dialectique, entre la forme prédéterminée et celle à construire, qui permet de pénétrer au cœur de l'œuvre.

Bien que brève, cette citation - placée par son auteur en exergue d'un chapitre consacré à une proposition de classification des compositions carnatiques suivie de leur brève description - souligne aussi l'apparente monotonie structurelle du répertoire compositionnel de l'Inde du Sud. Les différentes compositions reposent en effet, dans leur grande majorité, sur un même schéma de base et leur distinction concerne davantage leur destination, la nature de leur texte poétique que leur originalité structurelle. Cette ossature consiste en une succession de trois sections $\left(\right.$ anga $\left.^{26}\right)$ principales ayant les unes par rapport aux autres certains liens organiques. Ces trois parties sont appelées respectivement pallavi, anupallavi et caranam (cf. encadré).

Cette citation semble donc témoigner du peu d'intérêt que la pensée musicale carnatique prête à la forme en tant que telle, du moins lorsqu'elle cherche à isoler la composition de son interprétation. Or ces deux dimensions ne peuvent guère être détachées l'une de l'autre en Inde du Sud. Sans doute cela explique-t-il la maladresse, voire l'embarras, des musiciens et des musicologues indiens lorsqu'ils abordent l'œuvre comme des analystes travaillant à la table, c'est-à-dire en ne tenant compte que de son aspect composé, « achevé».

Les grands musiciens de la tradition carnatique ont souvent préféré s'exprimer de manière spontanée, faisant jaillir l'œuvre musicale - voire le texte et la musique - en l'improvisant. Tel fut invariablement le cas de Tyāgarāja. Ses improvisations ont été préservées par ses disciples, oralement ou par écrit, et transmises de génération en génération. La réflexion formelle ne se situe donc que rarement (cas des compositions pédagogiques surtout) a priori de l'acte créatif. Elle est avant tout, et particulièrement dans le krti, le fait de l'interprète et de l'interprétation.

\section{Le mot, la prière (sens de la forme)}

Même lorsque son degré de maturation le rend désirable, le fruit du manguier n'apporte que désappointement lorsqu'il n'a pas de jus. C'est pourquoi ce sont des poètes secs, ceux qui s'appuient seulement sur le sens des mots (Bansat-Boudon 1992: 97).

Comme la quasi-totalité des formes qui composent le répertoire carnatique, le krti est destiné d'abord à la voix, même s'il peut être interprété seulement par des instruments. Bien que nombre de musicologues, et même certains musiciens indiens, le considèrent comme secondaire, le texte poétique y tient une place essentielle. Le krti peut être parfois - de plus en plus souvent aujourd'hui - joué et apprécié comme une œuvre close. Cependant, cette tendance à ne l'appréhender que sous cet unique aspect en corrompt hélas, la plupart du temps, l'esprit et, par voie de conséquence, la forme. La ferveur du sentiment dévotionnel qui imprègne le poème disparaît alors, souvent au profit de la seule virtuosité technique. L'interprétation se cantonne à réaliser la composition, à ne 
plus improviser - ou du moins fort peu ou mécaniquement. C'est donc aussi le discours musical, en tant qu'élément intégrant du message, qui se trouve altéré. 


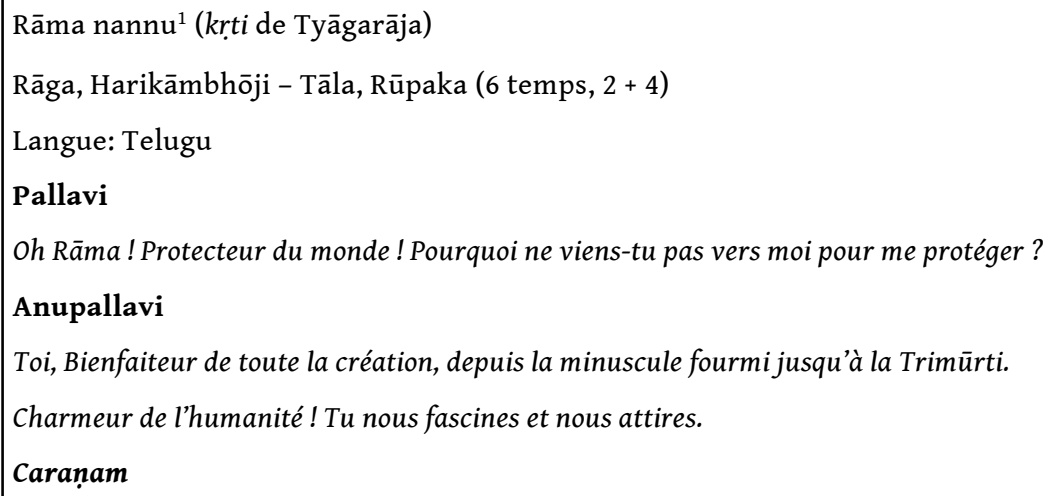

\section{Pallavi (sangati)}

;P, M, G, I ; R, S, ; R, G, ; ; II - - Ra - ma - Na- -- nnu- Bro--- va- ra- ----

,M P, M, MG | ; R, MGRS R, G, ; ; | - - Ra- ma- Na- -- nnu- Bro--- va- ra- ----

,M P, M, GM | PMGR MGRS R, G, ; ; | - - Ra- ma- Na- -- nnu- Bro--- va- ra- --. GMPD MDPP | MGR, MGRS R, G, ; ; || - - Ra- ma- Na- -- nnu- Bro--- va- ra- ---GMPD P, P,DP | MGR, G,MGRS R, G, ; ; || - - Ra- ma- Na- -- nnu- Bro--- va- ra- ----

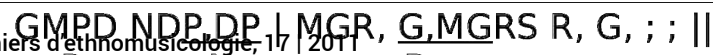
- Ra- ma-Na- -- nnu- Bro--- va- ra- ----

GMPD NSND | PMGR G,MGRS R, G, ; ; II - - Ra- ma- Na- -- nnu- Bro--- va- ra- -..-

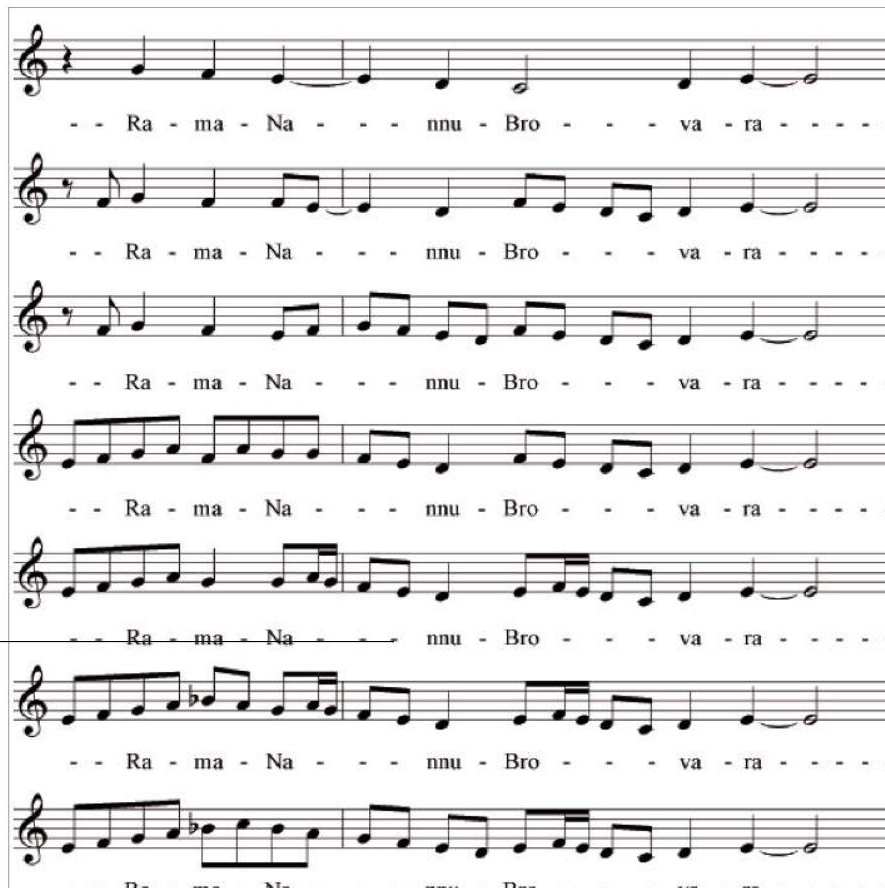



idéale implique nécessairement une " interprétation» (déduction et induction) de la part de son lecteur. L'interprétation du krti apparaît aussi comme un processus de présentification: manifestation de l'émotion qui en fut à la source, de la divinité qui l'a inspirée.

A travers le krti,l'interprète redevient à part entière un créateur. A travers son interprétation, le musicien - l'artiste et le dévot - parvient à manifester et parfois à atteindre le sentiment d'extase divine qui a inspiré son premier créateur ${ }^{29}$.

Tyāgarāja, s'il n'a pas été l'inventeur du krti, l'a en revanche porté à son point de perfection par la souplesse qu'il lui a donnée et l'introduction d'un procédé musical appelé sangati. Les sangati sont des variations musicales qui répètent une phrase du poème (généralement la première de chaque section) et qui suivent un processus progressif (additif) de développement. Ils peuvent apparaître dans chacune des trois parties du krti et sont, sauf rare exception, exclusivement liés à cette forme.

31 à sa louange mais surtout à la rendre présente, à dissoudre l'amour du dévot en elle - sont 
mises en valeur et nuancées à travers les sangati ${ }^{30}$. La principale raison d'être de ces variations, outre l'exacerbation graduelle du sentiment de ferveur que ces mentions induisent, consiste à éveiller l'esprit créatif de l'interprète, à l'inciter à participer, à s'immiscer au sein de la composition. En d'autres termes, le compositeur a légué à l'interprète ces modèles afin que celui-ci invente par lui-même d'autres variations (à ces endroits précis de la composition ou ailleurs). Ainsi les sangati sont-ils autant de pistes pour l'improvisation.

Stimuler le génie créatif de ses interprètes, les amener à chanter et ainsi à partager ce sentiment d'extase qu'elle contient en germe: tel est le but véritable du krtiti .

Fig. 4: Donner vie au kalaṃ. Vaikom, Kerala.

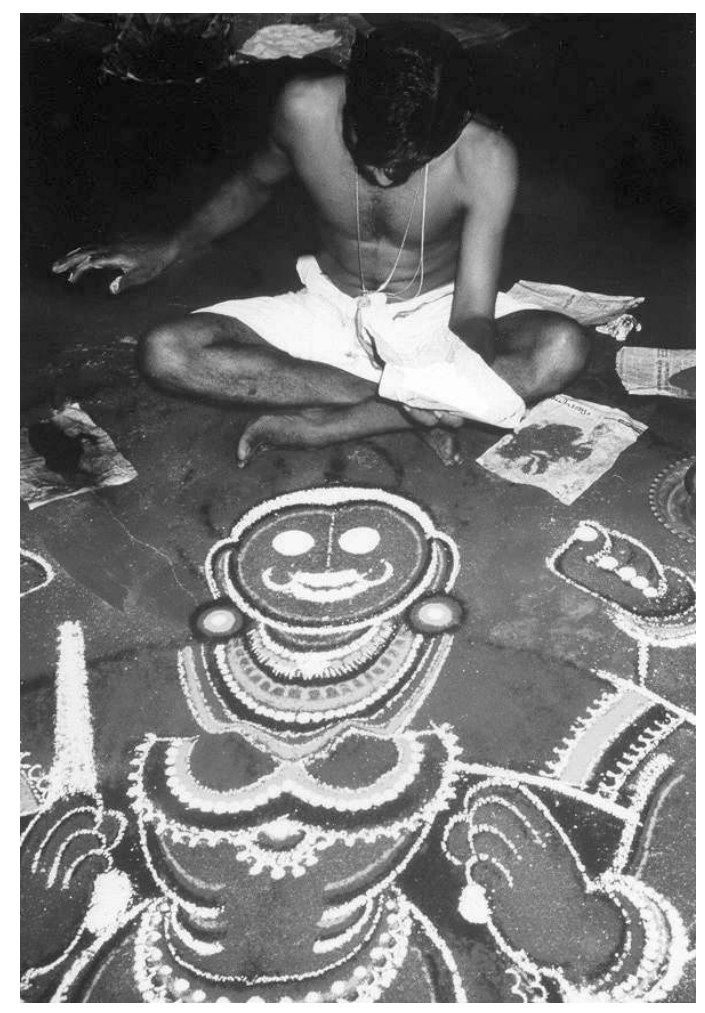

Photo: Marie-Danièle El Mouaffaq, 2000 souvent par ramifications - peut amener à considérer la composition comme un état transitoire de l'œuvre. L'œuvre vécue en est le fruit.

Les sangati improvisés, et plus largement l'improvisation, permettent au musicien de s'exprimer personnellement sur le plan technique, artistique. Ils apportent une dimension affective supplémentaire à la composition initiale, sans que celle-ci soit pour autant altérée par les sentiments individuels. Un dialogue, marqué musicalement par un jeu permanent entre la composition (dimension close de l'œuvre) et l'improvisation (son ouverture), s'instaure entre l'interprète et le compositeur. Cette véritable relation d'échange double celle du dévot et de la divinité.

Ce n'est que par le biais de l'improvisation que le krti atteint sa véritable dimension. Le krti représente par excellence l'œuvre à mettre en forme. L'interprétation vient parfaire ce qui a été donné par la composition ${ }^{33}$. 
34

en ce sens que le statut de la composition carnatique paraît souvent ambigu, voire contradictoire, pour un Occidental. Comme lui, et même davantage, le musicien, le mélomane, indien considèrent la composition comme le legs inestimable (ici, invariablement sacré) d'un créateur d'exception. Ainsi est-il concevable d'interpréter le krti littéralement, c'est-à-dire en suivant la partition à la lettre. Cependant, il n'en reste pas moins "perfectible». L'expression «améliorer la composition»" ${ }^{34}$ est même souvent employée par les musiciens et les musicologues indiens pour signifier qu'une interprétation sera parvenue à en retrouver le souffle initial. Dans ce contexte, la perfection de l'œuvre tient souvent davantage à celle de son expérience, qui est le jeu du composé et de l'improvisé, qu'à celle de son achèvement intrinsèque.

Tout comme la multiplicité des idoles, « l'œuvre revêt une multiplicité d'aspects à travers ses maintes possibilités d'interprétation. En utilisant la composition, les interprètes expriment aussi leur propre sentiment de bhakta» ${ }^{35}$.

carnatique a toujours accordé une place essentielle à l'improvisation dans le domaine musical et même poétique. Ainsi semble-t-il opportun de parler d' «amélioration» de la composition à propos d'une interprétation qui parvient à en ressentir puis à en révéler la vie intérieure, à « la faire revivre» telle qu'elle jaillit jadis de la voix même du compositeur. Celui-ci a exprimé avant toute chose un désir, une intention, de tout son être; les œuvres apparaissent presque secondaires et elles n'en sont en fait qu'une conséquence. La tradition dépend néanmoins de l'existence de ces individus d'exception car ils en ont fixé les modèles. La valeur humaine, artistique, religieuse de leurs créations a conduit leurs disciples, leurs auditeurs, à préserver une partie de ce qu'ils improvisaient. La vivacité de leur génie demeure et se transmet aussi, à travers les compositions, creusets présents et futurs de l'expression personnelle.

'il convient de parler de style (choix et expression personnels) au sein de la tradition carnatique, sur un plan purement musical du moins, c'est donc avant tout à propos de l'interprétation qu'il faut le faire plutôt que de la composition. A travers la « mise en forme» de l'œuvre, l'interprète laisse s'épancher (germer) ses sentiments, c'est-à-dire une partie de lui-même. Il importe néanmoins d'insister qu'il s'agit ici pour le musicien, comme dans l'ensemble du culte de la bhakti, de signifier une relation dévotionnelle sous un angle personnel et non pas de mettre en avant, encore moins d'« exhiber» sa propre individualité.

Le bhakta "doit apprendre à être dans le monde mais non pas du monde»

(Purandara Dāsa 1998: 104).

L'activité créatrice (et physique), souvent intense, du dévot n'est en ce sens qu'un moyen d'expression et d'imprégnation du sentiment divin. Les bhakta marchent de villes en villes, de temples en temples, à la manière des prophètes. Ils parlent, chantent, dessinent, utilisent tous les outils qui sont matériellement à leur disposition, et avec eux chacun de leur sens, pour manifester leur foi dans toutes ses dimensions et animer celle des autres hommes. Mais leur corps, comme les objets qui supportent leur croyance, ne vaut sur terre qu'en tant qu'allié de leur dévotion. L'expérience et l'action deviennent de merveilleux auxiliaires de l'accès à la « Délivrance» (mokșa), du moment où elles ne sont pas dirigées vers la satisfaction de l'ego.

De quelle utilité sont les yeux qui ne peuvent, à travers une pénétrante contemplation, comprendre la beauté du divin sommeillant sur le serpent sacré ? De quelle utilité peut être une telle façon de voir? De quelle utilité peut être le corps qui n'étreint pas passionnément celui de Sri Hari ${ }^{36}$, radieux comme les nuages de mousson? [...] De quelle utilité sont les mains qui n'adorent pas Sri Hari en lui 
lançant des fleurs de lotus et de jasmin [...] ? De quelle utilité est la langue qui ne chante pas la louange de Sri Rāma, le bienveillant protecteur de Tyāgarāja? (Tyāgarāja 1995: 524). couler comme l'huile que l'on verse sur la lampe». En chantant l'œuvre comme l'on attise la flamme de la lampe, l'interprète lui insuffle vie. Une part de sa propre existence, se dissout en elle. La forme excelle dans l'instant qui la fait exister. L'œuvre devient alors pour le musicien qui la pratique, au-delà de l'expression individuelle, un point de rencontre avec le compositeur, la tradition; son interprétation relie ce qui est passé à l'immédiateté. La composition, l'idole ne sont là que pour conduire à leur propre dépassement. La pratique est, outre une gustation esthétique, une expérience du divin en soi (une expérience du Soi) située au-delà des sens et des sentiments individuels.

Savourer [la saveur esthétique] n'est pas une activité de la langue mais une [activité] mentale. [...] C'est dans le monde seulement que cette [activité de gustation] s'accomplit comme la conséquence immédiate d'une activité de la langue (Bansat Boudon 1992: 108).

Expression spontanée de l'émotion intérieure du dévot, du compositeur et de l'interprète, le krti met davantage en valeur le sentiment du rāga (bhāva), que ses spécificités techniques ${ }^{37}$. De son interprétation émane parfois une saveur subtile: son rasa. Car si le krti est un support de la jubilation des sens, il est aussi une voie qui permet de s'en détacher. Le rasa - divin comme le silence qui succède à la dernière note - en est le fruit véritable, la " trace impalpable et secrète» ${ }^{38}$ que seuls les musiciens inspirés, ceux qui ont su pénétrer au cœur de la forme, parviennent à révéler.

\section{Un rituel du Kerala (dépasser la forme)}

Dans un petit temple du centre du Kerala, la grande fête se prépare. Kālī, « la Terrible», « la Brune " sera bientôt invoquée. Figures d'apparence, ses multiples représentations, violentes, meurtrières, sanguinaires, demandent à être dévoilées. Mais cette perception subtile passe d'abord par celle d'une éclatante et polymorphe épiphanie. Comme si la déesse ne parvenait à révéler au fidèle sa véritable essence, protectrice, maternelle, qu'après l'avoir rassasié en tous lieux, en tous sens. Et tous les sens humains seront aussi tour à tour sollicités. Par le dessin, la sculpture, la poésie, le chant puis la danse, la divinité sera mise en scène, mise en forme de multiples façons afin de la rendre toujours plus présente, toujours plus proche.

Le maître du rituel appelle Kāli: frénésie du tambour, les mains dessinent son image avec des poudres aussi légères que l'encens, le pinceau minutieux maquille le visage. Puis les chants semblent conquérir un autre espace, un autre temps, ceux de la déesse qu'ils font apparaitre aux rythmes vibrants des mots. Kāli se met à frémir; l'image plane du kalam ne parvient plus à contenir son énergie.

Le dessinateur, maintenant métamorphosé en Kālīi, semble émerger du kalạn. Les multiples incarnations de la déesse encerclent de toute part le croyant qui, situé au coeur de la forme divine, parviendra à percevoir sa bénéfique énergie. La danse, que prolongent les sons, devient aussi prise de possession, plus encore une rencontre entre le croyant et son dieu.

43 J'assistais, insatiable, conquis, à la cérémonie que j'avais commandée. La carte de l'Inde, comme l'image de Kālĭ, dessinée sur la pierre du temple, semblait avoir gagné en épaisseur, en profondeur aussi. 


\section{BIBLIOGRAPHIE}

BANSAT-BOUDON Lyne, 1992, Poétique du Nātyaśastra. Paris: Ecole Française d'Extrême-Orient.

BERTRAND Daniel, 2001, La musique carnatique, guide d'écoute de la musique classique de l'Inde du Sud. Paris: Editions du Makar.

BIARDEAU Madeleine, 1995, L’Hindouisme. Paris: Flammarion.

BRUGUIERE Philippe, 1994, La délectation du rasa, in Cahiers de Musique Traditionnelles 7: 3-26. Genève: Ateliers d'ethnomusicologie.

CONTRI Fabrice, 2001, « Les métamorphoses du rāga», in Analyse Musicale 39: 47-56.

ECO Umberto, 1965, L'œuvre ouverte. Paris: Editions du Seuil.

ESNOUL Anne-Marie, 1980, «Bhakti» in Encyclopcedia Universalis. Paris: E.U.

GOVINDA RAO T. K., 1997, Compositions of Muddusvāmi Dīkshitar. Chennai: Gānamandir Publications.

KRISHNAMŪRTHY S. V., 2000, Know Your Thyagaraja. vol. 1. Chennai: T.R. Publications.

PARTHASARATHY T. S., 1994, "The Kriti Form in Carnatic Music», in The Journal of the Music Academy. vol. LXV. Chennai: The Music Academy Madras.

PESCH Ludwig, 1999, The Illustrated Companion to South Indian Classical Music. Oxford: Oxford University Press.

PURANDARA DĀSA , 1985, « Ughabhogas», in S.K. Rāmachandra Rao: Purandara Sahitya Darshana. vol. 3. Bangalore: The Directorate of Kannada and Culture, Government of Karnataka.

PURANDARA DĀSA, 1998, « Ughabhogas», « Irabeku illadirabeku», in William J. Jackson: Songs of Three Great South Indian Saints, Calcutta, Chennai, Mumbai: Oxford University Press.

RĀGHAVAN V., 1966 [1981], The spiritual heritage of Tyagaraja. Mylapore, Chennai: Sri Ramakrishna Math Printed Press.

RĀMĀNUJAN A.K., 1999, « Hymns for the Drowning», in Indira Menon: The Madras Quartet. Lotus Collection. New Delhi: Roli Books.

SAMBAMOORTHY P., 1976, South Indian Music. vol. 3. Madras: The Indian Music Publishing House.

SUNDARAN S. P., 1987, « Tyagaraja and Nada Brahma» in Sruti, Aug/Sept: 36.

TALLOTTE William, 2003, Inde du Sud. Periya mêlam. Temple de Chidambaram. 1 CD Ocora / Radio France, C 560178.

TARA BĀLAGOPAL, 1998, Pancha Ratña Kritis of Saint Thyagaraja New Delhi: Harman Publishing House.

TYĀGARĀJA, 1995, « Nā Jvādhāra», « Ēlāvatāremettu Koṇțivi», « Intanuku vaṛnimpa», « Endu kaugalindurā», « Smaraṇē sukhamu», Ennaga manasuku rāni», « Rāma nannu brōvarā»,in T. K. Govinda Rao: Compositions of Tyāgarāja. Chennai: Gānamandir Publications.

TYĀGARĀJA, 1966 [1981], « Mērusāmāna dhīra», in V. Raghavan: The spiritual heritage of Tyagaraja. Mylapore, Chennai: Sri Ramakrishna Math Printed Press. 


\section{Références discographiques pour le krti « Rāma nannu brōvarā»}

SUBRAMA IAM Dr L., 1990, Anthologie de la musique classique de l'Inde du Sud (CD 2, plage 1).4 CD

Ocora C 59001-004

CONTRI Fabrice, 2004, Inde du Sud, O. K. Subramaṇiam, Nāgasvaram (plage 2). 1 CD INEDIT W 260119.

\section{NOTES}

1. Le point souscrit se prononce i. Ainsi krti se prononce kriti.

2. Ville du Tamil Nādu, située à la pointe méridionale du sous-continent indien.

3. Trimūrti (sanskrit) de tri (trois) et mūrti (matière, forme mais aussi manifestation, individu, représentation, statue, image, idole). Dans l'hindouisme, triple manifestation divine: Brahmā, Śiva, Viṣnu.

Les trois grands saints, compositeurs et poètes d'Inde du Sud (cf. note 23), nés au même endroit (Tiruvārūr, près de Thañjāvūr, Tamil Nādu) et à peu d'années de distance, sont souvent considérés dans la tradition carnatique (musique de l'Inde du Sud) comme l'incarnation musicale humaine de la Trimūrti et pour cette raison communément nommés « Trinité carnatique».

4. Dieu à tête d'éléphant dont le véhicule est une souris (mūṣa). muṣ- renvoie aussi à dérober, ravir.

5. bhakti (sanskrit): dévotion, adoration, amour; ferveur, hommage, fidélité; extase.

6. Dévot, adepte de la bhakti.

7. Dessin à base de poudres colorées réalisé sur le sol.

8. layam (sanskrit): absorption dans, attachement à; dissolution, mort.

9. Communication orale de Uṇnikrișṇan Mārār, acteur de muțiyêtțu, Kerala.

10. «Dérivé de la racine bhaj, partager, bhakti désigne en Inde la sorte de dévotion qui fait participer le fidèle à la personne et à l'essence de la divinité qu'il adore» (Esnoul 1980: 235-236). La bhakti apparaît également comme un choix: celui de la forme divine par le dévot. Le bhakta vouera durant toute son existence une constante dévotion à cette forme. La représentation divine, n'est qu'un état, une limitation d'un unique Absolu - les limites étant imposées par la forme elle-même.

11. ișta devatā (sanskrit) de ișta (désiré, désirable, aimé; choisi, préféré) et devatā (divinité, déité, idole).

12. Rāma, «le Charmant», héros du Rāmayaṇa, prince de la ville d'Ayōdhya, vénéré comme la septième incarnation (avatāra) de Viṣnu.

13. Communication orale de $S$. Rāmānujan, écrivain, metteur en scène, Thañjāvūr, Tamil Nādu.

14. Communication orale de P. N. Rāghavakuruppe, chanteur de sōpāna sangita, Vaikom, Kerala. La musique comme le théâtre, arts de mouvement, est une «Poésie qui s'incarne, de la Poésie animée» (Bansat-Boudon 1992: 99). Musique et danse prolongent le mouvement intérieur du corps, né de la jubilation de la foi.

15. Purandara Dāsa signait ses œuvres par Purandara Vittala.

16. Etres maléfiques, nocturnes, grands amateurs de chair crue et qui peuvent prendre la forme qu'ils désirent pour tromper leurs victimes.

17. De la lignée de Raghu, roi de la lignée solaire dont Rāma est l'arrière-petit-fils. Rāghava est l'un des noms de Rāma.

18. krti (sanskrit): composition, œuvre, création; enchantement. De $k$ 
-, faire; produire, créer (faire exister), fabriquer. « Le terme 'kriti' en Sanskrit possède plusieurs acceptions. 'Yat kritam tat kritih'. Tout ce qui est fait est un kriti. Il peut signifier - fabrication, réalisation, exécution [d'une œuvre], action, acte, création, œuvre, composition. [...] il désigne également la magie, l'enchantement !» (Parthasarathy 1994: 99-104).

19. De śyāma (noir, brun, de couleur foncée - est utilisé également pour le teint de la peau, comme une qualité) sundara (beau, joli, plaisant, aimable). Cet emploi renvoie au krti de Tyāgarāja, Śyāmasundarānga, où la peau de Rāma, comme celle de Kṛ̣ṇa, est décrite«brune comme un nuage de mousson».

20. Tyāgarāja, aux dires de ses disciples et des historiens indiens de la musique, improvisait également la plupart de ses poèmes conjointement à la musique.

21. Dans la tradition carnatique, la plupart des musiciens signent leurs œuvres avec le nom de la divinité de leur ville natale, de leur divinité familiale, de leur iș̣a devatā. Par cette signature ( $m u d r a \bar{a})$, ils effacent ainsi leur identité civile. Muttusvāmi Dīkșitar signait ses œuvres de la mudrā, Guruguha; Śyāma Śāstri par Śyāmakṛṣna. Tyāgarāja en revanche garda son nom pour mudrāa, Tyāgarāja étant le nom donné à Śiva dans la ville natale du compositeur, Tiruvārūr (le grand temple de cette ville est dédié à cet aspect du Dieu, « Seigneur de l'ascèse»).

22. « Nom donné au principe éternel qui anime l'individu empirique» (Biardeau 1995: 288-289).

23. Gōpuram : porte monumentale des temples de l'Inde du Sud.

24. Ville du Tamil Nādu.

25. svara (sanskrit): pris ici dans son acception de note de musique.

26. anga (sanskrit): membre; partie du corps; le corps; la personne, la forme; partie, portion.

27. Communication orale de O.K. Subramaniam, joueur de nādasvaram (hautbois), Kerala.

28. La partition est en cela une "notation ouverte» qui, bien qu'elle ne transcrive pas toute l'œuvre sonore, renvoie cependant à l'ouverture formelle de cette dernière.

29. O.K. Subramaniam.

30. Ces passages enrichissent l'œuvre tant sur le plan musical que littéraire. En ce domaine, le rôle du sangati consiste davantage à manifester, par la musique pure, l'essence du texte (le plus souvent expression de la foi) que de mettre «physiquement» en valeur les mots. Si la structure globale de chaque sangati, en tant que phrase musicale, se soumet en effet toujours à la structure syntaxique du discours littéraire, le mot en revanche, en tant qu'entité distincte de la globalité de la phrase, se plie entièrement aux contingences musicales. Ainsi l'élément matériel du texte - le signifiant - est-il progressivement dissout dans les entrelacs des rythmes, des lignes mélodiques et des « ornements». Cependant, il serait faux de considérer, que le texte devient un élément secondaire. Le signifié prévaut sur le signifiant et le poème, par le prisme du discours musical, acquiert une dimension expressive que les mots seuls ne sauraient lui donner. L'émotion musicale extrait l'essence subtile, la dimension indicible du langage parlé.

31. O.K. Subramaniam.

32. Le rāga est souvent considéré comme une graine que l'interprète habile et inspiré parvient à faire germer. De même dans le krti, l'improvisation naît, jaillit de la composition.

33. O.K. Subramaniam.

34. « to improve».

35. Mavelikara R. Prabhakara Varma.

36. Viṣnu, « Celui-qui-enlève (les péchés)».

37. Cet aspect plus technique est dévolu au varnam, une forme de composition particulièrement savante. Dans le var varnam, forme close, le compositeur fixe un modèle du rāga. L'improvisation $\mathrm{y}$ est absente ou très réduite.

38. "Le bhavarasa est la trace impalpable, secrète du rāga, le don subtil de la musique», communication orale de S. Rāmānujam. 


\section{RÉSUMÉS}

La forme occupe une place centrale en Inde tant dans le domaine de la pensée qu'au sein des différentes activités religieuses, artistiques et quotidiennes. Point de rencontre entre l'intellect, le corps et l'esprit, elle est aussi une voie. En elle se condensent la présence des dieux et la foi ardente des hommes. Multiple, elle est à l'image des aspects du divin et du regard, des sentiments des dévots. Elle offre également aux sens terrestres le support et le moyen de leur dépassement. L'œuvre musicale, tout comme l'idole - limitation formelle de l'Absolu et de sa quête - demande à être admirée, vénérée mais plus encore " pratiquée». Le répertoire de la tradition carnatique a su répondre à cette nécessité de manière originale notamment à travers une forme particulière de composition appelée krti dont Tyāgarāja fut le grand artisan. Ce saint-poète-musicien de l'Inde du Sud, inlassable dévot de Rāma, a trouvé dans cette forme musicale un moyen sans pareil pour exprimer, sous un angle personnel, sa ferveur et sa divinité d'élection tout en laissant ouvert un champ infini de lectures et d'interprétations possibles.

\section{AUTEUR}

\section{FABRICE CONTRI}

Fabrice Contri est né en 1969 à Phnom-Penh. Conjointement à l'étude de la musique classique occidentale (CNR de Boulogne-Billancourt et CNSM de Paris ; Université Sorbonne-Paris IV), il se consacre aux musiques traditionnelles et notamment à celles de l'Inde du Sud (recherches sur les musiques classiques et rituelles; pratique de la flûte carnatique comme instrument de rencontre et de compréhension). Après avoir été, pendant plus de quatre ans, professeur chargé de la programmation au CNR de Boulogne-Billancourt et avoir enseigné l'ethnomusicologie dans ce conservatoire, il est actuellement professeur au CNSM de Lyon et chargé de cours à l'Université (UCO) d'Angers. 